\title{
Dynamics of an electric current-carrying string loop near a Schwarzschild black hole embedded in an external magnetic field
}

\author{
Arman Tursunov, ${ }^{1,2,3, *}$ Martin Kološ, ${ }^{4, \dagger}$ Bobomurat Ahmedov, ${ }^{1,2,3, *}$ and Zdeněk Stuchlík ${ }^{4, \S}$ \\ ${ }^{1}$ Institute of Nuclear Physics, Ulughbek, Tashkent 100214, Uzbekistan \\ ${ }^{2}$ Ulugh Begh Astronomical Institute, Astronomicheskaya 33, Tashkent 100052, Uzbekistan \\ ${ }^{3}$ Max-Planck-Institut für Gravitationsphysik, Albert-Einstein-Institut, 14476 Golm, Germany \\ ${ }^{4}$ Institute of Physics, Faculty of Philosophy and Science, Silesian University in Opava, \\ Bezručovo nám.13, CZ-74601 Opava, Czech Republic
}

(Received 7 January 2013; published 3 June 2013)

\begin{abstract}
We study motion of an electric current-carrying string loop oscillating in the vicinity of the Schwarzschild black hole immersed in an external uniform magnetic field. The dependence of boundaries and different types of motion of the string loop on magnetic field strength is found. The dynamics of the string loop in the Cartesian $x y$ plane depends both on value and direction of the magnetic field and current. It is shown that the magnetic field influence on the behavior of the string loop is quite significant even for weak magnetic field strength. The oscillation of the string loop becomes stronger or weaker in dependence on the direction of the Lorenz force. We illustrate the various regimes of the trajectories of the string loop that can fall down into the black hole, escape to infinity, or be trapped in the finite region near the horizon for the different representative values of the magnetic field. We have also considered the flat spacetime limit as the condition of the escape of the string loop from the neighborhood of the black hole to infinity. We found the expression for the magnetic field strength for which the oscillatory motion of the string loop totally vanishes and the string loop can have maximal acceleration in the perpendicular direction to the plane of the loop.
\end{abstract}

DOI: 10.1103/PhysRevD.87.125003

\section{INTRODUCTION}

Current carrying strings moving axisymmetrically along the axis of a Kerr black hole [1], a Schwarzschild-de Sitter black hole [2,3], or a braneworld black hole or naked singularity [4] could represent a toy model of plasma that exhibits associated stringlike behavior via dynamics of the magnetic field lines in the plasma [5-8] or due to thin isolated flux tubes of plasma that could be described by an one-dimensional string [9]. Tension of such a string loop prevents its expansion beyond some radius, while its world sheet current introduces an angular momentum barrier preventing the loop from collapsing into the black hole. Such a configuration was also studied in $[10,11]$. It has been proposed in [1] that the current carrying string can be used as a model for relativistic jet formation around the black hole and in [3] that it can model high-frequency quasiperiodic oscillations in the vicinity of the black hole horizon.

In our preceding recent paper [3], the possibility to convert motion of a string loop originally oscillating around a black hole in one direction to the perpendicular direction, modeling thus an accelerating jet, has been studied. It is well known that due to the chaotic character of the motion of string loops such a transformation of the energy from the

\footnotetext{
*armantursun@gmail.com

†martin.kolos@fpf.slu.cz

*ahmedov@astrin.uz

§zdenek.stuchlik@fpf.slu.cz
}

PACS numbers: 11.27.+d, 04.70.-s, 04.40.-b, 04.25.-g

oscillatory to the linear mode is possible $[1,2,10]$ and in the preceding paper [3] we have estimated its efficiency and studied the role of the cosmic repulsion, that can be, surprisingly, relevant in astrophysical phenomena [4].

Here we extend our previous research to the case when the black hole is embedded in the magnetic field and investigate the motion of an axisymmetric electric current-carrying string loop in the background of the Schwarzschild black hole and external, uniform, axisymmetric magnetic field. There exist both theoretical and experimental indications that a magnetic field may be present in the vicinity of black holes. A regular magnetic field can exist near a black hole surrounded by conducting matter (plasma), e.g., if the black hole has an accretion disk. The magnetic field near a stellar mass black hole may contain a contribution from the original magnetic field of the collapsed progenitor star. The dynamo mechanism in the plasma of the accretion disk might generate a regular magnetic field inside the disk. Such a field cannot cross the conducting plasma region and is trapped in the vicinity of the black hole (see, e.g., discussion in Frolov [12]).

Stellar mass and supermassive black holes often have jets that are collimated fluxes of relativistic plasma. It is generally believed that the magnetohydrodynamics of the plasma in strong magnetic and gravitational fields of the black holes would allow one to understand formation and energetics of the black hole jets [1]. The magnetic field in the vicinity of the black holes might play an important role in transfer of the energy from the accretion disk to jets. 
Existence of a regular magnetic field near black holes is also required for the proper collimation of the plasma in the jets.

For the estimation of the magnetic field strength at the horizon radius of Schwarzschild black holes, we use the magnetic coupling process in dependence on the black hole mass $M_{\mathrm{BH}}$ and the accretion rate $\dot{M}$ performed in [13] and based on the use of the fundamental variability plane for stellar mass black holes, active galactic nuclei and quasistellar objects. The variability plane can be presented in the following form

$$
\log \nu_{b}=\log \dot{M}-2 \log M-14.7,
$$

where $\dot{M}$ is the accretion rate in $\mathrm{g} / \mathrm{s}, M=M_{\mathrm{BH}} / M_{\odot}$ is the black hole mass in $M_{\odot}$, and $\nu_{b}$ is the break frequency in $\mathrm{Hz}$. The magnetic field strength is derived in a result of the magnetic coupling (MC) process which is responsible for the interaction between the black hole and its surrounding accretion disk.

The MC process provides the relation between the magnetic field strength at the horizon of Schwarzschild black hole $B_{H}$ and its mass $M$ and the accretion rate $\dot{M}$. This relation is based upon the balance between the pressure of the magnetic field at the horizon and the accreted matter pressure of the innermost part of an accretion flow:

$$
B_{H}=\frac{1}{R_{H}} \sqrt{2 k_{m} \dot{M} c},
$$

where $R_{H}=2 G M_{\mathrm{BH}} / c^{2}$ is the radius of the Schwarzschild black hole. $k_{m}$ is a magnetization parameter indicating the relative power of the $\mathrm{MC}$ process with respect to the disk accretion. If the $\mathrm{MC}$ process dominates over the disk accretion, $k_{m}>1$, and if it is dominated by latter, $k_{m}<1$. The case $k_{m} \approx 1$ corresponds to equipartition between these two processes. At logarithm scaling, this can be written in the form

$$
2 \log B_{H}=\log \dot{M}-2 \log M+2 \log f\left(k_{m}\right),
$$

where

$$
f\left(k_{m}\right)=\frac{c^{2} \sqrt{2 k_{m} c}}{2 G M_{\odot}} \cong 0.83 \sqrt{k_{m}} .
$$

Equations (1) and (3) gave together

$$
B_{H} \approx 0.83 \sqrt{\nu_{b} k_{m}} \times 10^{7.35} .
$$

It was shown in paper [14] that the break frequency $\nu_{b}$ is determined by both the black hole mass $M_{\mathrm{BH}}$ and the accretion rate $\dot{m}_{\text {Edd }}=\dot{M} / \dot{M}_{\text {Edd }}$ as follows:

$$
\nu_{b}=0.029 \varepsilon \dot{m}_{\mathrm{Edd}}\left(10^{6} \frac{M_{\odot}}{M_{\mathrm{BH}}}\right),
$$

where $\varepsilon$ is the efficiency of the mass to energy conversion (usually one assumes $\varepsilon=0.1$ ). Using equations stated above, one can estimate the strength of the magnetic field in the vicinity of stellar mass and supermassive black holes as

$$
\begin{aligned}
& B \approx 10^{8} \mathrm{G}, \quad \text { for } M \approx 10 M_{\odot}, \\
& B \approx 10^{4} \mathrm{G}, \quad \text { for } M \approx 10^{9} M_{\odot} .
\end{aligned}
$$

Charged particle motion in electromagnetic fields surrounding the black hole has been studied in different works (see e.g., $[15,16])$. Off-equatorial motion of charged particles in background gravitational and electromagnetic fields has been studied in papers $[17,18]$. Detailed analysis of the astrophysics of rotating black holes in the presence of electromagnetic fields in connection with energetic mechanisms such as the Penrose process has been discussed by Wagh and Dadhich in paper [19]. The Blandford-Znajek mechanism applied for the black hole with a toroidal electric current was investigated in papers [20,21]. Observational evidences of the existence of a magnetic field around black holes can be found in papers (see, e.g., $[22,23])$.

In this paper, we study dynamics of electric currentcarrying string loop in the magnetic field around the black hole. To make our discussion more concrete, we use the evaluation for the magnetic field given in papers $[13,15]$. Namely, the characteristic scales of the magnetic field $B$ are of the order of $B_{1} \sim 10^{8} \mathrm{G}$ near the horizon of a stellar mass $\left(M_{1} \sim 10 M_{\odot}\right)$ black hole, and of the order of $B_{2} \sim$ $10^{4} \mathrm{G}$ near the horizon of a supermassive $\left(M_{2} \sim 10^{9} M_{\odot}\right)$ black hole. We use the estimates above to describe only the domain of the physical parameters, which characterize our system, and to define the validity of the adopted approximations. Let us notice that both the fields $B_{1}$ and $B_{2}$ are weak in the following sense: The spacetime local curvature created by the magnetic field $B$ is of the order of $G B^{2} / c^{4}$. It is comparable to the spacetime curvature near a black hole of mass $M$ only if

$$
\frac{G B^{2}}{c^{4}} \sim \frac{1}{r_{g}^{2}} \sim \frac{c^{4}}{G^{2} M^{2}} .
$$

For a black hole of mass $M$, this condition holds if the strength of such a magnetic field satisfies the condition

$$
B \sim B_{M}=\frac{c^{4}}{G^{3 / 2} M_{\odot}}\left(\frac{M_{\odot}}{M}\right) \sim 10^{19} \frac{M_{\odot}}{M} \mathrm{G} .
$$

Evidently, the quantities $B_{1,2}$ for both the stellar mass and supermassive black holes presented above are much smaller than the corresponding $B_{M}$. In what follows we assume that the magnetic field is weak $B \ll B_{M}$ and its energy momentum does not modify the background black hole geometry. This means that for our problem the field $B$ can be considered as a test field in the given gravitational background. Such a magnetic field practically does not affect motion of neutral particles.

To characterize relative strength of magnetic and gravitational forces acting on an electric current-carrying string loop with mass $m$ and charge $q$ in the vicinity of a black hole of mass $M$ immersed in a magnetic field with strength $B$, one can use the following dimensionless quantity [12]: 


$$
b=\frac{|q| B G M}{m c^{4}} .
$$

However, hereafter we suppose that the string loop is massless and the dimensionless quantity $b$ being valid for the massive particle motion can be applied for the estimation of the efficiency of a magnetic field even for the massless string loop.

The influence of the magnetic field induced by the Lorentz force of the order of $q B /(m c)$ can be quite strong and non-negligible. The simple estimation of the quantity $b$ in the black hole environment gives [12,24]

$$
b \sim 4.7 \times 10^{6}\left(\frac{q}{e}\right)\left(\frac{m}{m_{\mathrm{p}}}\right)^{-1}\left(\frac{B}{10^{8} \mathrm{G}}\right)\left(\frac{M}{M_{\odot}}\right),
$$

where $e$ and $m_{\mathrm{p}}$ are the charge and mass of the proton, respectively. On the basis of this estimation, one can conclude that the effect of the magnetic field on the motion of such relativistic systems cannot be neglected even in weak magnetic field approximation.

Our investigation may be relevant for understanding of the phenomena of collimated jets which are exhibited in systems that range from accreting young stars, neutron stars, and black holes to supermassive black holes in active galactic nuclei and quasars.

The paper is organized as follows. Section II is devoted to study of the equations of string motion in the magnetic field background. In Sec. III the influence of the magnetic field on the oscillatory motion of the string loop in the flat spacetime has been considered. In Sec. IV the behavior of the string loop and relevant effects in the vicinity of the black hole immersed in an external magnetic field is studied. The concluding remarks and discussions are presented in Sec. V.

Throughout the paper, we use a spacetime signature as $(-,+,+,+)$ and a system of geometric units in which $G=1=c$. (However, for those expressions with an astrophysical application we have written the speed of light explicitly). Greek indices are taken to run from 0 to 3 .

\section{STRING LOOP MOTION IN THE COMBINED GRAVITATIONAL AND EXTERNAL MAGNETIC FIELD}

We study motion of an axisymmetric electric currentcarrying string loop threaded onto an axis passing through the symmetry center of the Schwarzschild black hole spacetime and chosen to be the $y$ axis in the presence of an external magnetic field which is also directed along the $y$ axis (see Fig. 1). The string loop can oscillate, changing its radius in the $x-z$ plane, while propagating in the $y$ direction. Assumed axial symmetry of the string loop allows one to investigate only one point on the loop because one point path can represent the whole string movement. The trajectory of the loop is then represented by a curve given in the $2 \mathrm{D} x-y$ plane. The string loop tension and the world sheet current corresponding to an angular momentum parameter form barriers governing its dynamics. These barriers are modified by the gravitational field of the Schwarzschild black hole characterized by the mass $M$ and by the external uniform magnetic field with the strength $B$.

The Schwarzschild spacetime metric in spherical coordinates reads

$$
d s^{2}=-f(r) d t^{2}+f^{-1}(r) d r^{2}+r^{2}\left(d \theta^{2}+\sin ^{2} \theta d \varphi^{2}\right),
$$

where the metric function

$$
f(r)=1-\frac{2 M}{r}
$$

is expressed through the total mass $M$ of the black hole.

Since the string loop preserves its axial symmetry during motion, it is obvious from Fig. 1 that the description of the trajectory of only one point of the string allows us to define full string loop motion [25]. Therefore according to [1,2] it is useful to use the Cartesian coordinates

$$
x=r \sin \theta, \quad y=r \cos \theta
$$

for the proper description of the string loop motion.

Then the $3+1$ dimensional Schwarzschild spacetime metric (13) with coordinates $(t, r, \theta, \varphi)$ can be rewritten in new coordinate notations $(t, x, y, \varphi)$ in the following $3+1$ dimensional form:

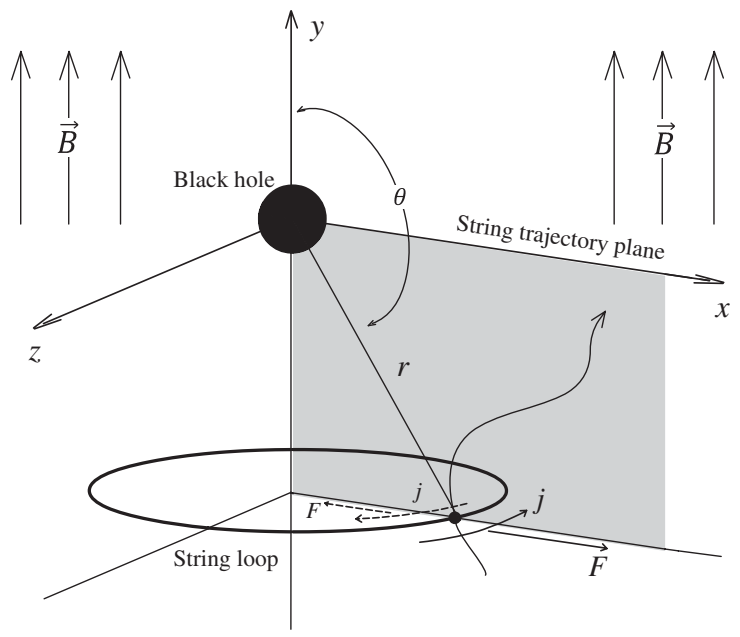

FIG. 1. Electric current-carrying string loop in external magnetic field moving around a Schwarzschild black hole. The behavior of only one point on the loop can describe all string movement. Then string trajectory is shown on the $x-y$ plot. In the presence of the electric current there is a Lorenz force acting on the string perpendicularly to the plane of magnetic field strength and electric current. The direction of the Lorenz force is "positive" when it acts on the string loop in the direction from the black hole to space (solid arrow). The Lorenz force is "negative" when it is directed into the black hole (dashed arrow). 


$$
\begin{aligned}
d s^{2}= & -f d t^{2}+x^{2} d \varphi^{2}+\frac{x^{2}+f y^{2}}{f\left(x^{2}+y^{2}\right)} d x^{2} \\
& +\frac{f x^{2}+y^{2}}{f\left(x^{2}+y^{2}\right)} d y^{2}+2 \frac{(1-f) x y}{f\left(x^{2}+y^{2}\right)} d x d y,
\end{aligned}
$$

where the characteristic function of the line element is taken in the form

$$
f(x, y)=1-\frac{2 M}{\sqrt{x^{2}+y^{2}}} .
$$

According to Wald [26] the existence of the spacetime Killing vectors $\xi^{\alpha}$ which satisfy the Killing equation

$$
\xi_{\alpha ; \beta}+\xi_{\beta ; \alpha}=0
$$

gives us the right to write the solution of vacuum Maxwell equations $\square A^{\mu}=0$ for the vector potential $A_{\mu}$ of the electromagnetic field in the Lorentz gauge in the form

$$
A^{\alpha}=C_{1} \xi_{(t)}^{\alpha}+C_{2} \xi_{(\varphi)}^{\alpha} .
$$

Because of the asymptotic properties of spacetime (13) at the infinity the integration constant $C_{1}=0$ whereas the second integration constant $C_{2}=B / 2$. Consequently, the magnetic field potential will take the following simple form [26]:

$$
A^{\alpha}=\frac{B}{2} \xi_{(\varphi)}^{\alpha},
$$

where commuting Killing vector $\xi_{(\varphi)}=\partial / \partial \varphi$ generates rotations around the symmetry axis. The nonvanishing covariant component of the 4-vector potential of the electromagnetic field will take the form

$$
A_{\varphi}=\frac{B}{2} r^{2} \sin ^{2} \theta=\frac{B}{2} x^{2} .
$$

The magnetic field is uniform at infinity where it has the strength $B \geq 0$.

Next, we summarize the equations of string motion in the Hamiltonian approach $[25,27]$ in the presence of the magnetic field. The string world sheet is described by the spacetime coordinates $X^{\alpha}\left(\sigma^{a}\right)$ given as functions of two world sheet coordinates $\sigma^{a}$ (with $a=0,1$ ). We adopt coordinates $(\tau, \sigma)$ as the parameter $\tau$ denoting an affine parameter related to the proper time measured along the moving string and parameter $\sigma$ reflecting the axial symmetry of the oscillating string.

The dynamics of the string is described by the action related to the string tension $\mu>0$ and a scalar field $\varphi$ as $\varphi_{, a}=j_{a}$ determines current (angular momentum) of the string $[1,2]$. The assumption of axisymmetry implies the scalar field in linear form with constants $j_{\sigma}$ and $j_{\tau}$ :

$$
\varphi=j_{\sigma} \sigma+j_{\tau} \tau
$$

It can be viewed as a $1+1$ dimensional massless radiation fluid, with positive energy density and equal pressure, i.e., tension. In a general situation, integration of the equations of motion of string loops or open strings is a very complex task that has to be treated by numerical methods only, and has been discussed in a variety of papers [10,11,28-36].

It has to be emphasized that the string loop itself is the additional source of the electric and magnetic field. The magnetic field created by the azimuthal current in a current loop in the Schwarzschild and Kerr spacetimes has been considered in papers [37,38]. Following $[39,40]$ and using the Cartesian coordinates with $r=\sqrt{x^{2}+y^{2}}$, we find that a current-carrying string loop located at the equatorial $\theta=\pi / 2$ plane, symmetrically around a black hole with mass $M$ at radius $r_{s}$ has nonvanishing components of the magnetic field in the region $r \geq r_{s}$ expressed in the form

$$
\begin{aligned}
B^{\hat{x}}=\frac{3 \mu x y}{4 M^{2} r^{3}}\left[\frac{2 \ln f}{\sqrt{f}(1+\sqrt{f})}-(1+f)\left(1+\frac{1}{\sqrt{f^{3}}}\right)+4\right], \\
B^{\hat{y}}=-\frac{3 \mu}{4 M^{2} r^{3}}\left[\frac{2 \ln f}{1-f}\left(y^{2}+\frac{x^{2}}{\sqrt{f}}\right)\right. \\
\left.+(1+f)\left(\frac{x^{2}}{\sqrt{f^{3}}}-\frac{3-f}{1-f} y^{2}\right)\right]
\end{aligned}
$$

where $f=1-2 M / r$ is the characteristic function of the line element called lapse function. It is identical to the magnetic field of a dipole with moment

$$
\mu=\pi r_{s}^{2} I\left(1-\frac{2 M}{r_{s}}\right)^{1 / 2},
$$

where $I$ is the total electric current. The components of the magnetic field (23) and (24) taken in the asymptotics, i.e., $f \rightarrow 1$ or $M / r \rightarrow 0$ reduce to the flat-space form

$$
\begin{gathered}
B^{\hat{x}}=\frac{3 \pi x y}{r^{5}} I r_{s}^{2}, \\
B^{\hat{y}}=\frac{\pi\left(2 y^{2}-x^{2}\right)}{r^{5}} I r_{s}^{2} .
\end{gathered}
$$

In terms of the string loop given by [1,2] current $I$ corresponds to $j_{\sigma}=J \Omega$. Then the norm of the magnetic field of the current-carrying string loop in arbitrary point with $r \geq r_{s}$ can be written as

$$
B_{s}=\frac{\pi \sqrt{x^{2}+4 y^{2}}}{\left(x^{2}+y^{2}\right)^{2}} J \Omega r_{s}^{2} .
$$

Clearly, $B_{s}$ is maximal when $r_{s}=r=\sqrt{x^{2}+y^{2}}$ and decreases with the increase of $r_{s}$. For the parametrization taken in papers [2,3], one can estimate the typical value of the magnetic field of the string loop with radius $r_{s}=10$, angular momentum $J / \sqrt{\mu}=11$, and the maximal current parameter $\Omega=1$ as

$$
B_{s} \approx 1.34 \times 10^{-3} \mathrm{G} \text {. }
$$


For the parametrization taken above, we used the limiting tension for the Nambu-Goto string model of the cosmic strings [41] as $\mu=1.5 \times 10^{-7}$. Its contribution to the energy of the string which is proportional to $B_{s}^{2}$ is negligibly small in comparison with those of the external magnetic field which is estimated as $\sim 10^{8} \mathrm{G}$ for stellar mass black holes and $\sim 10^{4} \mathrm{G}$ for supermassive black hole. On the other hand, in the following we will not neglect the interaction of the external and self-magnetic fields which leads to the appearance of the rotating moment which is proportional to the electric current of the string and external magnetic field $B$. In vector form a rotating moment can be written as

$$
\vec{M} \sim J \Omega \vec{B} r_{s}^{2} .
$$

This implies that in the presence of an electric current in the string loop there exists a force which tries to hold the string perpendicularly to the vector of the strength of the ambient magnetic field (i.e., in the $x$-z plane) while the string loop can freely propagate in the $y$ direction. Therefore the selfmagnetic field of the string loop appears in the expression for the effective energy (38) as an interaction with the ambient magnetic field rather than a free term of order $B_{s}^{2}$.

The symmetry of the Schwarzschild spacetime and the assumption of the axisymmetric string oscillations enables a substantial simplification and the string motion can be treated using the Hamiltonian formalism. Following [27], one can introduce the Hamiltonian (further we choose a system of units with string tension $\mu=1$ )

$$
\begin{aligned}
2 H= & g^{\alpha \beta} P_{\alpha} P_{\beta}+Q+x^{2}+2\left(j_{\tau}^{2}+j_{\sigma}^{2}\right) \\
& +\frac{\left[2\left(j_{\tau}^{2}-j_{\sigma}^{2}\right)-Q\right]^{2}}{4 x^{2}},
\end{aligned}
$$

where $P_{\mu}=p_{\mu}+q A_{\mu}$ is the generalized 4-momentum of the string loop and parameter $Q$ denotes

$$
Q \equiv A_{\varphi}\left(2 \sqrt{2} j_{\sigma}+A_{\varphi}\right) .
$$

Equation (31) with the above-mentioned statements means that the string loop can be described by the usual Hamiltonian of a point particle of charge $q \sim j_{\tau}$ propagating in the four-dimensional curved spacetime and electromagnetic background given by the vector potential $A_{\mu}$.

To parametrize the solution, one can introduce new variables of the current components:

$$
J^{2} \equiv j_{\sigma}^{2}+j_{\tau}^{2}, \quad \omega \equiv-j_{\sigma} / j_{\tau} .
$$

The minus sign in the definition of $\omega$ is chosen in order to obtain correspondence of positive angular momentum and positive $\omega$. Note that $-j_{\sigma} / j_{\tau}=j^{\sigma} / j^{\tau}$. $J$ determines the angular momentum parameter of the string.

Because of the spherical symmetry of the spacetime we can assume $J>0$ in the following, while $\omega$ can be both positive and negative. The case with $\omega=0$ corresponds to the stable current-carrying loop [27]. The current $j_{\sigma}$ can be expressed in terms of $J$ and $\omega$ as

$$
j_{\sigma}^{2}=\frac{J^{2} \omega^{2}}{\omega^{2}+1} .
$$

Two spherical coordinates $t$ and $\varphi$ allow one to find the constants of motion. We use the existence of two conserved quantities associated with the Killing vectors:

$$
\begin{gathered}
E=-\xi_{(t)}^{\mu} P_{\mu}=-P_{t}, \\
L=\xi_{(\varphi)}^{\mu} P_{\mu}=-2 j_{\tau} j_{\sigma}+q A_{\varphi} .
\end{gathered}
$$

\section{A. Effective potential of the motion}

In the spherically symmetric spacetime (13) using Eqs. (21) and (33)-(36) the Hamiltonian can be expressed in the form

$$
H=\frac{1}{2} f(r) P_{r}^{2}+\frac{1}{2 r^{2}} P_{\theta}^{2}-\frac{E^{2}}{2 f(r)}+\frac{V_{\mathrm{eff}}}{2 f(r)},
$$

where

$$
V_{\mathrm{eff}}=f(r)\left\{\frac{B^{2} x^{3}}{8}+\frac{\Omega J B x}{\sqrt{2}}+\left(1+\frac{J^{2}}{x^{2}}\right) x\right\}^{2}
$$

is an effective potential for the string motion in a magnetic field in Cartesian coordinates, $B$ is the strength of the external magnetic field and a new dimensionless parameter $\Omega$ :

$$
\Omega \equiv \frac{\omega}{\sqrt{1+\omega^{2}}}
$$

varies in the range $-1<\Omega<1$.

The first term in the right-hand side of effective potential (38) is responsible for the pure contribution of the external magnetic field background to the "effective" energy because the density of the magnetic field energy is proportional to $B^{2}$ and the space volume to $x^{3}$.

The second term in the right-hand side of effective potential (38) is responsible for the interaction between the electric current carried in the string loop and the external magnetic field. This term can be associated as the interaction of two magnetic fields, where one of them is "global" external magnetic field $\sim B$, and another one is the "local" self-magnetic field of the string loop $\sim J \Omega / x$, according to (34) and (39). The sign of this term can be either positive or negative depending on the sign of $\Omega$, i.e., the direction of the electric current carrying on the loop. $\Omega$ is positive if the direction of the vector of the self-magnetic field strength coincides with the direction of the vector of the strength of the external magnetic field and negative if these vectors are directed in opposite sides. Since the direction of the external magnetic field is given as an initial condition, the direction of the Lorenz force acting on the string is determined by the direction of the current. The last term in the right-hand side of effective potential (38) corresponds to the contribution to the effective energy produced by the current (angular momentum) and the 
tension of the string loop. This term coincides with those of the paper [2] which is obtained for the nonmagnetic case and electric-neutral string.

Hereafter for some representative plots we will consider only three different values of $\Omega=-1 / \sqrt{2}, 0,1 / \sqrt{2}$ related to the boundaries of motion. The case $\Omega=0$ (i.e., $\omega=0$ ) corresponds to the null electric current while $\Omega= \pm \frac{1}{\sqrt{2}}$ corresponds to $\omega= \pm 1$. The sign of the parameter $\Omega$ depends on the choice of the direction of the electric current. In accordance with [2], one may call $\Omega$ as an electric current parameter, $J$ as an angular momentum parameter (or string parameter), and $B$ as the magnetic field parameter or magnetic parameter. Notice that in contrast to the case when there is no magnetic field [2], here, the Hamiltonian depends on parameter $\Omega$ together with $J$ and $B$.

\section{B. Boundary energy function}

The condition $H=0$ which determines the regions allowed for the string motion [25] can be written in the form

$$
E^{2}=\dot{r}^{2}+f(r) r^{2} \dot{\theta}^{2}+V_{\text {eff }} .
$$

The loci where the string loop has zero velocity $(\dot{r}=0$ and $\dot{\theta}=0$ ) form boundary of the string motion. We can define the boundary energy function by equating it to the effective potential:

$$
E_{\mathrm{b}}^{2}=V_{\text {eff }}
$$

In the Cartesian coordinates one can get the relation

$$
E_{\mathrm{b}}(x, y, J, B, \Omega)=\sqrt{f(r)} g(x),
$$

where $r=r(x, y)=\sqrt{x^{2}+y^{2}}$ and

$$
g(x)=\frac{B^{2} x^{3}}{8}+\frac{\Omega J B}{\sqrt{2}} x+\left(1+\frac{J^{2}}{x^{2}}\right) x .
$$

The function $f(r)$ reflects the spacetime properties, while $g(x)$ is responsible for the interaction of the charged string loop with magnetic field. The behavior of the boundary energy function of the string motion is given by the interplay of the functions $f(r)$ and $g(x)$. Assuming that the string loop is at rest initially, i.e., assuming $\dot{r}(0)=0$ and $\dot{\theta}(0)=0$, the initial position of the string can be located at some point of the energy boundary function $E_{\mathrm{b}}(x, y)$ of its motion. Notice that the term $g(x)$ is independent of the coordinate $y$, which enters only the spacetime term $f(x)$

Since we use the Cartesian coordinates it is easy to find the local extrema of the boundary energy function $E_{\mathrm{b}}$ determined by the relations

$$
\begin{gathered}
x f_{r}^{\prime} g(x)+2 f(r) r g_{x}^{\prime}=0, \\
g(x) y f_{r}^{\prime}=0,
\end{gathered}
$$

where the prime ()$_{m}^{\prime}$ denotes the derivative with respect to the coordinate $m$.

\section{MOTION IN THE FLAT SPACETIME LIMIT AND ESCAPE CONDITION}

We use the following procedure in order to determine the condition whether the oscillating string escapes to infinity. The string loop can escape to infinity if its energy is bigger than the rest energy at infinity or when $E>E_{\text {flat }}$ and the escape is determined by the limiting energy determined in the flat spacetime. It is easy to find conditions for the boundary energy function for the flat spacetime taking the characteristic function of the line element in the form

$$
f(r)=1
$$

and the energy boundary function in flat spacetime takes the simple form

$$
E_{\text {flat }}(x)=g(x) .
$$

Therefore, the energy tends to the infinity when $x \rightarrow 0$, i.e., $E_{\text {flat }}(x \rightarrow 0) \rightarrow \infty$, and the energy tends to the zero when $x \rightarrow \infty$, i.e., $E_{\mathrm{flat}}(x \rightarrow \infty) \rightarrow \infty$. In the presence of a magnetic field, a minimum of the function (47) always exists (for $B>0, J>0$ and $-1<\Omega<1$ ) that is located at

$$
x_{\mathrm{flat}}^{\min }=\sqrt{\frac{2 \sqrt{2}}{3 B^{2}}\left(\sqrt{3 B^{2} J^{2}+2 K^{2}}-\sqrt{2} K\right)},
$$

where

$$
K \equiv 1+\Omega J B / \sqrt{2} .
$$

In the absence of a magnetic field (i.e., for $B=0$ ) the minimum of the energy of the string loop in the flat spacetime will be located at

$$
x_{\text {flat }}^{\min }=J,
$$

and takes the value

$$
E_{\text {flat }}=2 J
$$

Profiles of the energy boundary function for the flat spacetime are given in Fig. 2 where we compare the case $B=0, \Omega=0$ with those $B>0, \Omega=0$ and $B>0, \Omega \neq 0$. As one can see from Fig. 2 the presence of the negative current reduces the minimum of the oscillatory energy which means that in such conditions the string loop will have the strongest acceleration in the $y$ direction. Hereafter in the figures for the energy of the string loop the curves corresponding to the flat spacetime are indicated by dashed curves.

In accord with the result of the string loop motion in the Schwarzschild spacetime [2], one can conclude that the string loop can escape to infinity if its energy is bigger than 


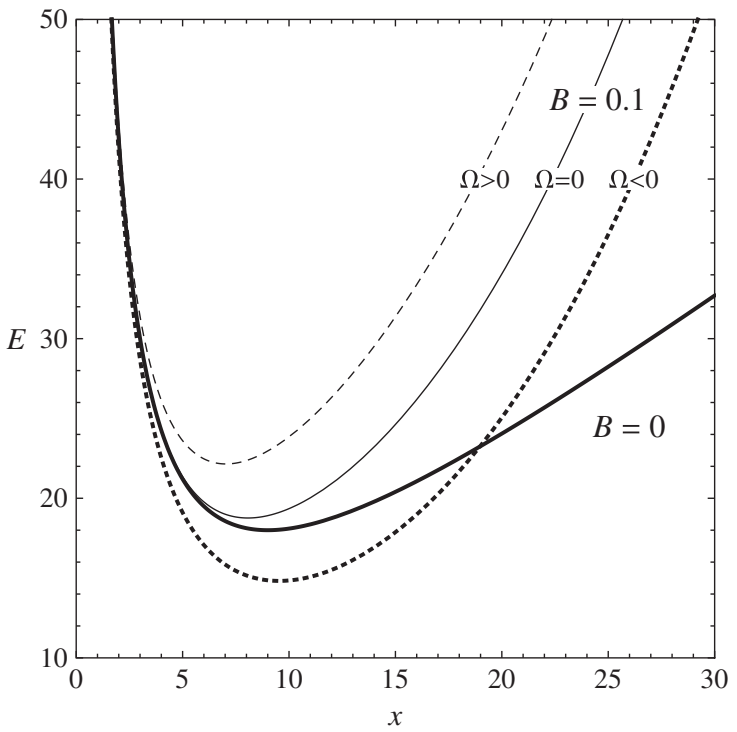

FIG. 2. Influence of the magnetic field and the electric current on the $x$ profile of the energy for the fixed value of the angular momentum parameter $J=9$. The case of zero magnetic field is indicated by a solid thick curve while the case of nonzero magnetic field $B=0.1$ is shown for the following three representative values of the electric current parameter: $\Omega=+\frac{1}{\sqrt{2}}$ is dashed, $\Omega=-\frac{1}{\sqrt{2}}$ is dotted, and $\Omega=0$ is a solid thin.

the rest energy at infinity, i.e., when $E>E_{\text {flat }}$, given by the condition (47). If the energy is equal or lower than the rest energy (47), the string loop cannot escape and must be trapped in a finite region above the horizon or captured by the black hole. The regions of trapped oscillations are most extended at the equatorial plane, when $y=0$ and shrink with an increase of the value of $y$. When $y \rightarrow \infty$ the region reduces to the flat line according to the expressions (47) and (48).

According to $[1,2]$, the oscillatory energy of the string loop in the background of black hole spacetime can be transmitted into the kinetic energy of the translational motion of string along the $y$ axis and inversely the translational energy can be converted into the energy of string oscillations. This phenomenon is called transmutation effect. As indicated in paper [3] in the absence of a magnetic field this effect completely works only in one direction since there remains inconvertible energy of string loop $E_{0(\min )}=2 J$ being the constant of motion (when $J>0$ ). But in the presence of a uniform magnetic field the situation is completely different and the string oscillatory energy $E_{x}$ can be lowered for the appropriately defined values of the motion parameters.

In order to find a complete solution of this problem and find the influence of magnetic field to this effect we first consider the decomposition of the full energy of string loop in the flat spacetime.

Decomposition of the energy of string loop (40) in the flat spacetime $(f(r)=1)$ in the Cartesian coordinates at the presence of magnetic field gives

$$
E^{2}=\dot{y}^{2}+\dot{x}^{2}+g(x)^{2}=E_{y}^{2}+E_{x}^{2},
$$

where the $x$ and $y$ modes of energy are denoted in the form

$$
\begin{gathered}
E_{y}^{2}=\dot{y}^{2}, \\
E_{x}^{2}=\dot{x}^{2}+\left[\frac{B^{2} x^{3}}{8}+\frac{\Omega J B}{\sqrt{2}} x+\left(1+\frac{J^{2}}{x^{2}}\right) x\right]^{2} .
\end{gathered}
$$

The $x$ mode of full energy $E_{x}$ is minimal at the location given by Eq. (48). Then neglecting higher orders of the magnetic parameter $B$ (since we work in weak magnetic field), the minimal value of the energy $E_{x}$ can be written in the following form:

$$
E_{x(\min )}=2 J+\frac{J^{2} \Omega B}{\sqrt{2}}+\frac{1}{8}\left(1-\Omega^{2}\right) J^{3} B^{2} .
$$

In the absence of a magnetic field (i.e., when $B=0$ ) this expression turns out to the condition $E_{0}=2 J$ which coincides with Eq. (25) from the paper [3], where there is no possible transmutation between $x$ and $y$ energy modes. However, in the presence of magnetic field, with the increasing of the magnetic parameter $B$, the energy $E_{x}$ decreases while $E_{y}$ remains constant. For the critical value of the magnetic parameter $B$,

$$
B_{\text {crit }}=\frac{2 \sqrt{2}\left(\Omega+\sqrt{3 \Omega^{2}-2}\right)}{J\left(\Omega^{2}-1\right)},
$$

the $x$ mode of the energy $E_{x(\min )}=0$, i.e., oscillations of string loop vanish at the location $x_{\text {flat }}^{\text {min }}$ given by the expression (48). Then the total amount of oscillatory energy can be transmitted into the translational energy. Note that

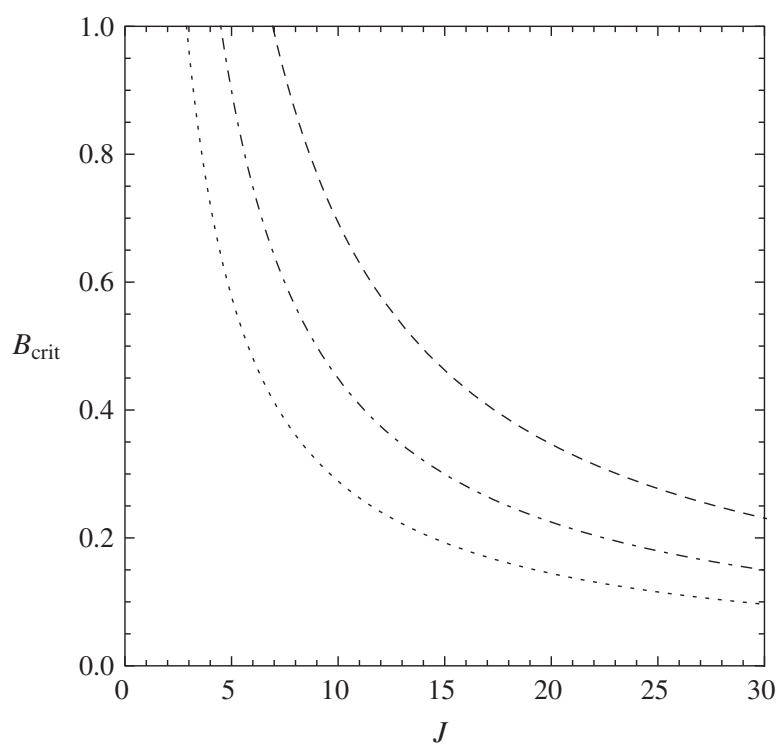

FIG. 3. Critical values of the magnetic parameter $B$ in dependence on $J$ for three representative values of electric current parameter: $\Omega=-0.82$ is dashed, $\Omega=-0.85$ is dot-dashed, and $\Omega=0.99$ is dotted. 
such a critical magnetic field can be defined only for negative values of the current parameter in the region $-1<\Omega \leq-\sqrt{2 / 3}$. The dependence of the critical magnetic field on the current is shown in Fig. 3.

\section{MOTION IN THE SCHWARZSCHILD SPACETIME}

\section{A. Equations of the motion}

The characteristic function of the line element for the Schwarzschild spacetime takes the following form:

$$
f(r)=1-\frac{2 M}{r} .
$$

Introducing an affine parameter of the string motion $\zeta$, the Hamilton-Jacobi equations

$$
\frac{\mathrm{d} X^{\mu}}{\mathrm{d} \zeta}=\frac{\partial H}{\partial P_{\mu}}, \quad \frac{\mathrm{d} P_{\mu}}{\mathrm{d} \zeta}=-\frac{\partial H}{\partial X^{\mu}}
$$

applied to the Hamiltonian (37) imply an equation of motion in the form

$$
\begin{gathered}
\dot{r}=f P_{r}, \\
\dot{P}_{r}=\frac{1}{f} \frac{P_{\theta}^{2}}{r^{4}}\left(f r-\frac{1}{2} \frac{\mathrm{d} f}{\mathrm{~d} r} r^{2}\right)-\frac{\mathrm{d} f}{\mathrm{~d} r} P_{r}^{2}-\frac{1}{f} \frac{\mathrm{d} V_{\mathrm{eff}}}{\mathrm{d} r}, \\
\dot{\theta}=\frac{P_{\theta}}{r^{2}}, \quad \dot{P}_{\theta}=-\frac{1}{f} \frac{\mathrm{d} V_{\text {eff }}}{\mathrm{d} \theta},
\end{gathered}
$$

where the dot denotes the derivative with respect to the affine parameter $\zeta$ as $\dot{F}=\mathrm{d} F / \mathrm{d} \zeta$.

\section{B. Boundaries of motion in dependence on the magnetic field}

Analysis of the boundaries of motion allows us to classify the types of dynamics and determine their dependencies on the magnetic field and other parameters of the string loop. If the string motion is given by initial conditions $(\dot{r}=0$ and $\dot{\theta}=0)$ and nonvanishing angular momentum $(J \neq 0)$ then the string energy can be calculated from the energy boundary function $E=E_{b}(J)$. The Schwarzschild geometry introduces a characteristic length scale corresponding to the radius of the black hole horizon $r_{\mathrm{h}}=2 M$. We restrict our consideration to the region above the black hole horizon, $r>r_{\mathrm{h}}$. In the Schwarzschild spacetime the function $E_{\mathrm{b}} \rightarrow+\infty$ when $r \rightarrow \infty$, but $E_{\mathrm{b}} \rightarrow 0$ when $r \rightarrow 2 M$.

It is more convenient to introduce the dimensionless coordinates taking them in the form

$$
x \rightarrow \frac{x}{M}, \quad y \rightarrow \frac{y}{M}, \quad r \rightarrow \frac{r}{M},
$$

and introduce the normalized parameters of the angular momentum, energy, and magnetic field as

$$
J \rightarrow \frac{J}{M}, \quad E \rightarrow \frac{E}{M}, \quad B \rightarrow B M .
$$

Then the extrema equation (44) can be written in the form of a quintic equation in the $x$ coordinate

$$
\frac{3}{8} B^{2} x^{5}-\frac{5}{8} B^{2} x^{4}+\left(1+\frac{B J \Omega}{\sqrt{2}}\right)\left(x^{3}-x^{2}\right)-J^{2} x+3 J^{2}=0,
$$

while Eq. (45) gives us the solution $y=0$.

Assuming that the magnetic field in the given gravitational background is weak, one can write the extrema equation for angular momentum parameter $J$ as the expansion of the small-parameter $B$ in the form

$$
J=J_{\mathrm{E}}(x, B, \Omega)=\frac{x \sqrt{x-1}}{\sqrt{x-3}}+\frac{\Omega B x^{2}(x-1)}{2 \sqrt{2}(x-3)},
$$

where the first term in the right-hand side of Eq. (64) exactly coincides with Eq. (78) of paper [2] obtained for the nonmagnetic case while the second term in the righthand side of (64) is responsible for the contribution of the interactive magnetic field term on the extrema function $J$. In the following we assume $J>0$ due to the spherical symmetry of the spacetime.

Plots of the function $J_{E}(x)$ for the selected values of the magnetic parameter $B$ are presented in Fig. 4 and indicated by solid curves. However, in the presented figures we have used the original expression for $J$ without the expansion where the terms being proportional to $B^{2}$ in Eq. (64) were neglected. In the region above the black hole horizon the function $J_{\mathrm{E}}(x)$ diverges at the distance $x_{\mathrm{div}}=3$ similarly to the case of Schwarzschild spacetime in the absence of the external magnetic field.

The local extrema $x_{\min }$ of the function $J_{\mathrm{E}}$ can be found by equalization of the derivative with respect to $x$ of the function (64) to zero. The location $x_{\min }$ determines the marginally stable stationary position of string loops in the Schwarzschild spacetime and it is substantially lower in comparison with the innermost stable circular orbit radius. Then substituting the value of $x_{\min }$ into (64), one can obtain the minimal value of $J_{\mathrm{E}}$ :

$$
J_{\mathrm{E}}\left(x_{\min }\right)=J_{\mathrm{E}(\min )} \text {. }
$$

This position is interesting since the boundary energy function for the values of string parameter $J>J_{\mathrm{E}(\min )}$ has two extrema, i.e., maximum and minimum (see Fig. 5). For $J<J_{\mathrm{E}(\min )}$ there are no extrema of the energy boundary function above the black hole horizon $(x>2)$. For $J=J_{\mathrm{E}(\min )}$ the energy boundary function $E_{\mathrm{b}}(x, J)$ has an inflex point. Quantitative changes of the values $J_{\mathrm{E}(\min )}$ from the values of magnetic parameter $B$ for three different cases of current $\Omega$ are given in Fig. 6 .

Using the extrema equation (63) in the equatorial plane $y=0$, one can obtain both maxima and minima of the energy boundary function in dependence on $J$ and $B$ for the 


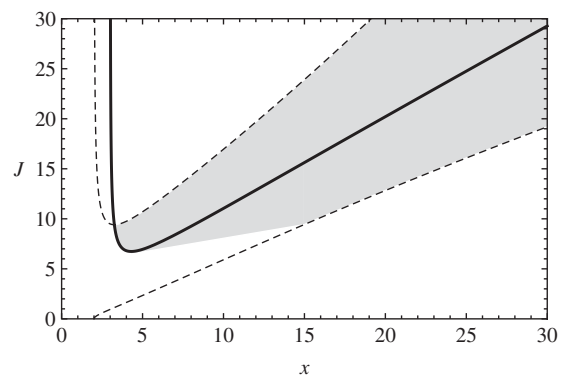

(a) $\Omega=-1 / \sqrt{2}, B=0.01$

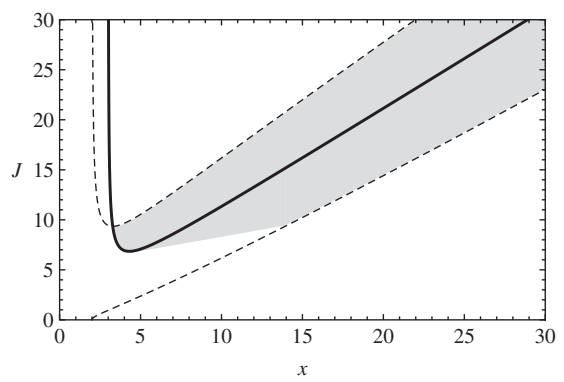

(d) $\Omega=0, B=0$

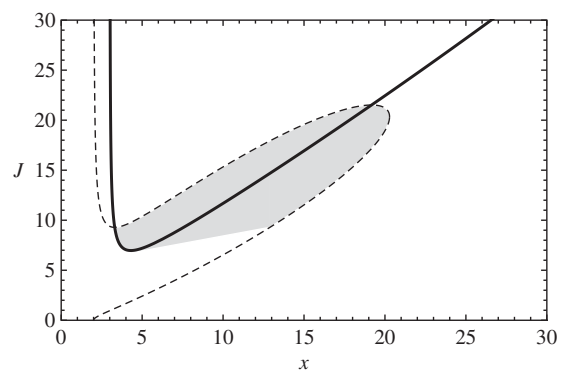

(g) $\Omega=1 / \sqrt{2}, B=0.01$

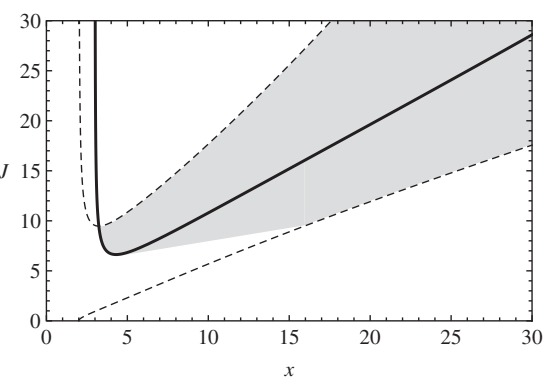

(b) $\Omega=-1 / \sqrt{2}, B=0.02$

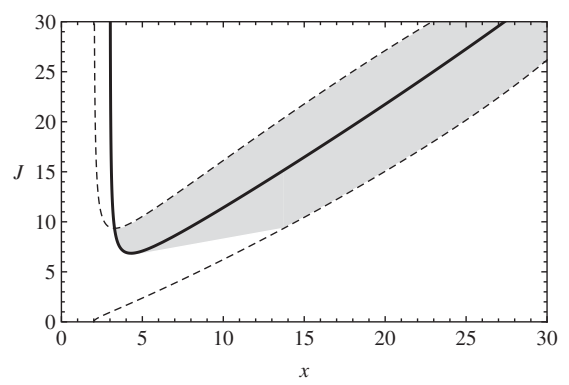

(e) $\Omega=0, B=0.02$

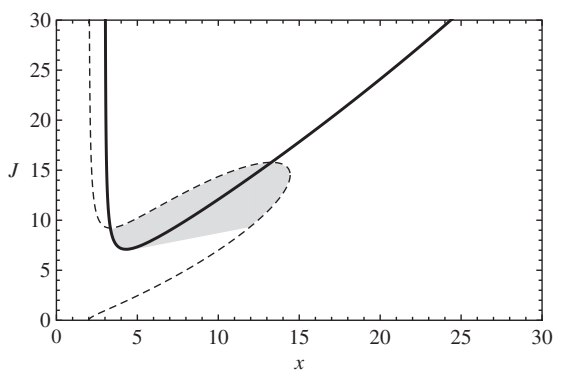

(h) $\Omega=1 / \sqrt{2}, B=0.02$

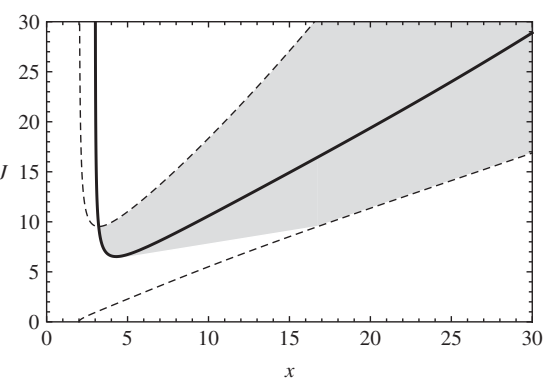

(c) $\Omega=-1 / \sqrt{2}, B=0.03$

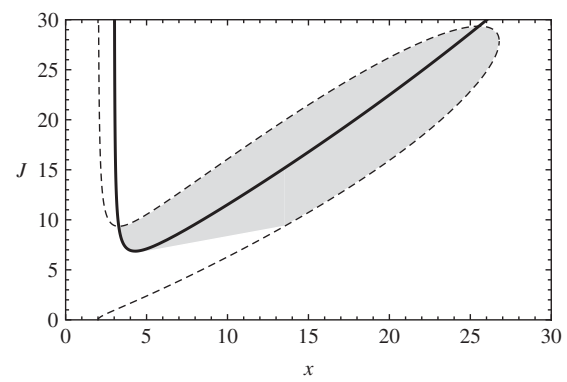

(f) $\Omega=0, B=0.03$

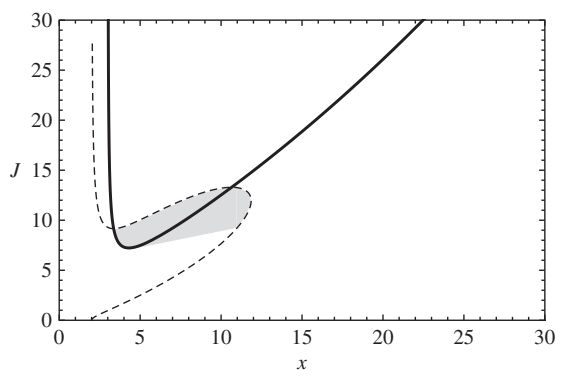

(i) $\Omega=1 / \sqrt{2}, B=0.03$

FIG. 4. String parameter function $J(x)$ for different representative values of the magnetic parameter $B$ and electric current parameter $\Omega$. Solid curves represent the extrema angular momentum (string parameter) $J_{E}$ as a function of the Cartesian coordinate $x$. The dashed curves correspond to the "lake" angular momentum functions for the various values of the parameter $B$ which are the solutions of Eq. (55) in the equatorial plane. These curves allow us to distinguish four different types of the behavior of the string loop motion. Regions where the trapped states can exist are shaded.

different values of the current parameter $\Omega$ as shown in Fig. 5. As one can see from the plots in the 2 nd and 3rd rows, an increase of the magnetic parameter $B$ leads to the narrowing of the energy boundaries, while in the 1 st row for the weak magnetic field the situation is quite different and boundaries of the energy (maximum and minimum) move away from each other for the negative values of the current parameter $\Omega=-1 / \sqrt{2}$. The oscillatory motion is allowed for the string loops with $J>J_{\mathrm{E}(\min )}$ and energy satisfying the condition $E_{\mathrm{b}(\min )}(J)<E<E_{\mathrm{b}(\max )}(J)$. String loops with $J<J_{\mathrm{E}(\min )}$, or with $E<E_{\mathrm{b}(\min )}(J)$ and $J>J_{\mathrm{E}(\min )}$ can be captured by the black hole.

The most interesting regions in Fig. 5 are zones between dashed and the lowest solid lines in which the string loop may neither escape to infinity nor be captured by the central black hole. These are so-called "trapped" states of the string loop motion. As one can see from the first row of Fig. 5 with the increase of magnetic field the trapped states of the loop decrease while the boundaries become wider. The situation in the second and third rows of Fig. 5 is opposite to the first row for the case of the weak magnetic field. In order to determine the conditions for trapped state existence we use the method developed in our preceding paper [2]. The trapped states of the string loop near the black hole correspond to "lakes" determined for appropriately chosen energy levels by the energy boundary function $E_{\mathrm{b}}(x, y, J, B, \Omega)$. First, the restrictions for the trapped oscillations in the $x$ direction have to be represented by "projected" angular momentum function $J_{\mathrm{p}}$ determined through the projection of the extremal (maximal) energy level $E_{\mathrm{b}(\max )}\left(x_{\max }, y, J, B, \Omega\right)$ onto the energy boundary function itself. It can be easily done with the help of the numerical simulation. Choosing the value of $J_{\mathrm{p}}$ for the fixed $y$, one can find the corresponding value of the maximum of the energy boundary function $E_{\mathrm{b}(\max )}\left(x_{\max }, y, J_{\mathrm{p}}, B, \Omega\right)$ and the related coordinate $x_{\mathrm{p}}$ where 


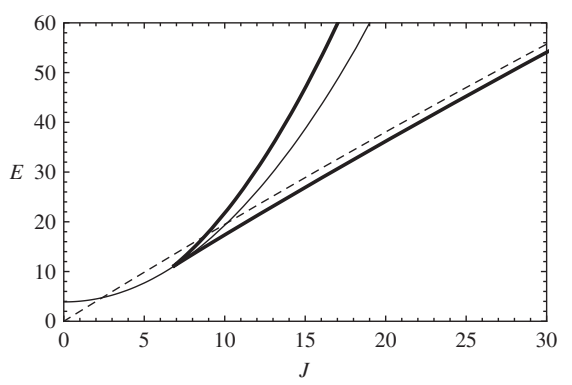

(a) $\Omega=-1 / \sqrt{2}, B=0.01$

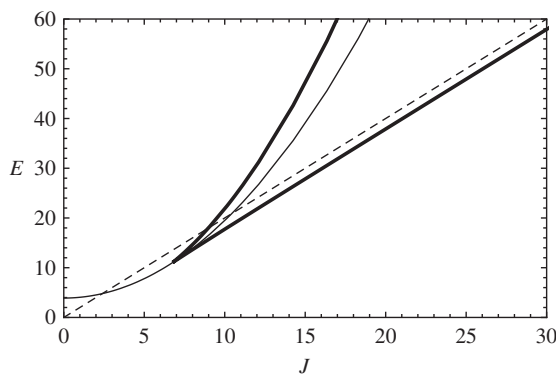

(d) $\Omega=0, B=0$

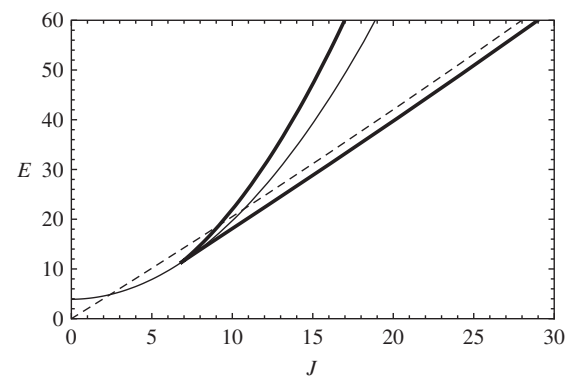

(g) $\Omega=1 / \sqrt{2}, B=0.01$

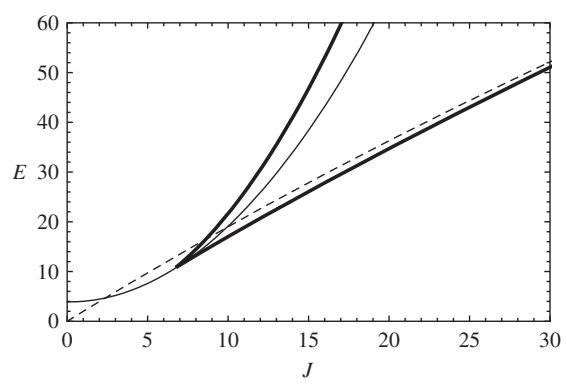

(b) $\Omega=-1 / \sqrt{2}, B=0.02$

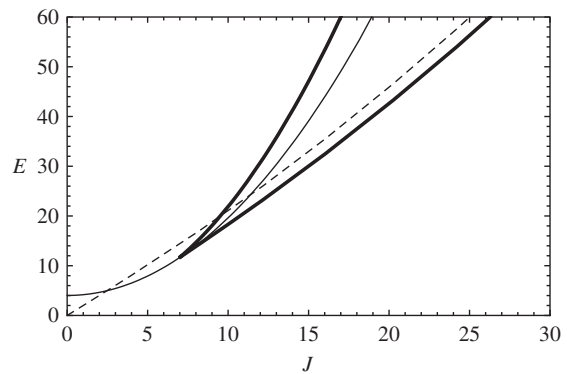

(e) $\Omega=0, B=0.1$

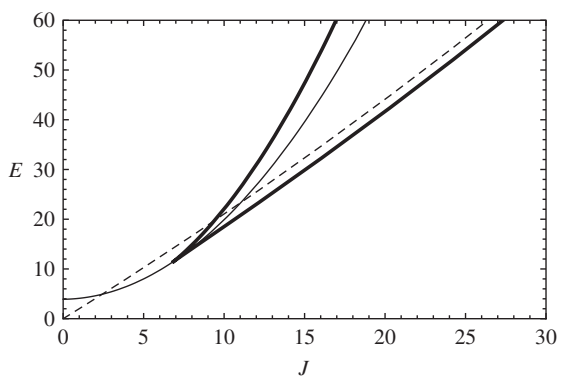

(h) $\Omega=1 / \sqrt{2}, B=0.02$

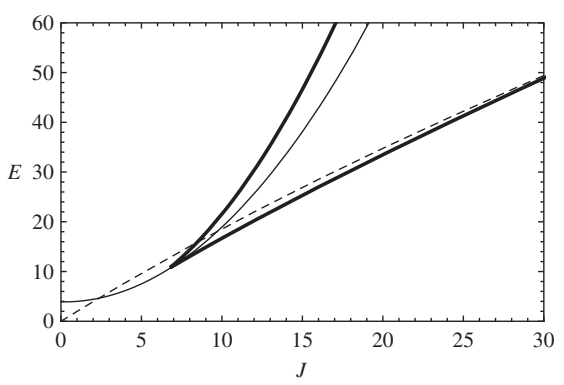

(c) $\Omega=-1 / \sqrt{2}, B=0.03$

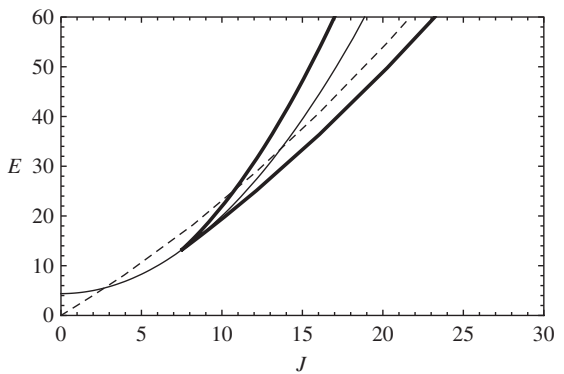

(f) $\Omega=0, B=0.2$

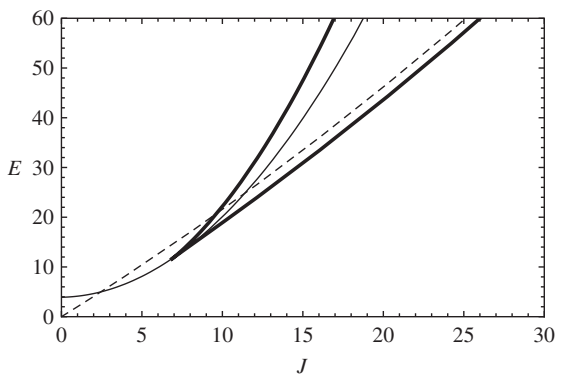

(i) $\Omega=1 / \sqrt{2}, B=0.03$

FIG. 5. Boundaries of the energy as a function of string parameter $J$ for different values of the magnetic parameter $B$ and electric current parameter $\Omega$. Thick solid curves correspond to the maxima and minima of the boundary energy function $E_{b}$ in Schwarzschild spacetime, while dashed curves represent the minimum of the boundary energy taken for the flat spacetime and given by (47). Thin solid line represents the energy $E_{\mathrm{b}}\left(x_{0}, y_{0}, J, B, \Omega\right)$ taken as an example at the point with coordinates $x_{0}=5, y_{0}=1$.

$$
E_{\mathrm{b}(\max )}\left(x_{\max }, y, J_{\mathrm{p}}, B, \Omega\right)=E_{\mathrm{b}}\left(x_{\mathrm{p}}, y, J_{\mathrm{p}}, B, \Omega\right) .
$$

Then we solve the equation

$$
E_{\mathrm{b}}(x, y, J, B, \Omega)=2 J+\frac{J^{2} \Omega B}{\sqrt{2}}+\frac{1}{8}\left(1-\Omega^{2}\right) J^{3} B^{2},
$$

where the expression in the right-hand side is the energy of the string loop in the flat spacetime $E_{\text {min }}^{\text {flat }}$ determined by Eq. (55). Consequently, the existence of the trapped states of the oscillating string loop are restricted by the condition

$$
J_{\mathrm{L} 1}(x, y)<J<J_{\mathrm{L} 2}(x, y),
$$

where the lake angular momentum functions $J_{\mathrm{L} 1}(x, y)$ and $J_{\mathrm{L} 2}(x, y)$ are solutions of Eq. (55). They are illustrated by the dashed curves in Fig. 4. The regions of the trapped states are shaded.

One of our main interests is to describe the influence of the magnetic field on the energy of the electric current-carrying string loop in the case when $\Omega$ is negative and the Lorenz force acting on the string decreases the value of the effective potential (38). One can easily find the location of the string

$$
x_{\mathrm{b}}=2 \sqrt{\frac{\sqrt{2}|\Omega| J}{B}}
$$

where the energy of the electromagnetic field is balanced by the Lorenz force energy and it is the position where the string loop has the same energy level as in the absence of a magnetic field.

For the loop positions $x<x_{\mathrm{b}}$, the Lorenz force energy will dominate the electromagnetic field energy and the bound string energy will be lower in comparison with that in the case without magnetic field. For the loop positions $x>x_{\mathrm{b}}$ the energy of the electromagnetic field will prevail the Lorenz force energy and the boundary energy of the string loop will be bigger in comparison with that in the 


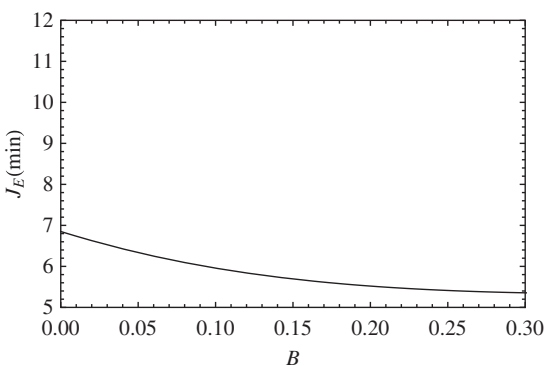

(a) $\Omega=-1 / \sqrt{2}$

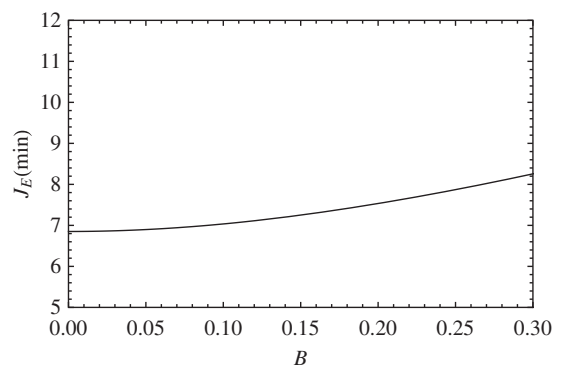

(b) $\Omega=0$

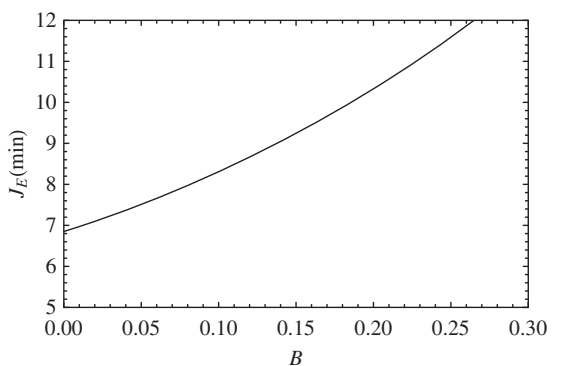

(c) $\Omega=1 / \sqrt{2}$

FIG. 6. Dependence of the local minimal value of string function $J_{\mathrm{E}}$ on the magnetic parameter $B$ for three representative values of parameter $\Omega$.

case without a magnetic field. This effect is relevant only for the negative values of the parameter $\Omega<0$ while for the positive values of the parameter $\Omega>0$ the boundary energy always grows with the increase of the magnetic field. It is interesting that the position (69) does not depend on the gravitational field properties and is valid for both
Schwarzschild and flat spacetimes. This means that the appearance of the specific space position in the magnetic background in which the string loop behaves exactly as in the nonmagnetized case is the property purely governed by the magnetic field. The position of the string loop $x=x_{\mathrm{b}}$ and its corresponding energy for the fixed angular

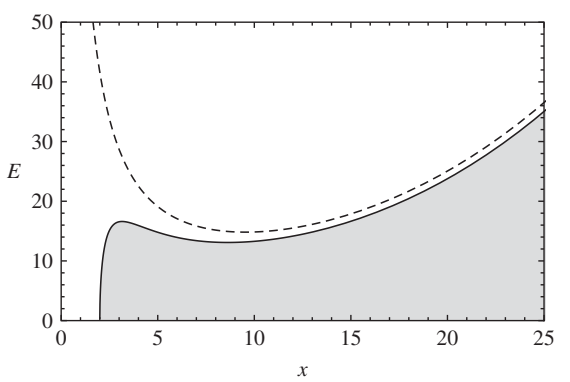

(a) $\Omega=-1 / \sqrt{2}, B=0.1$

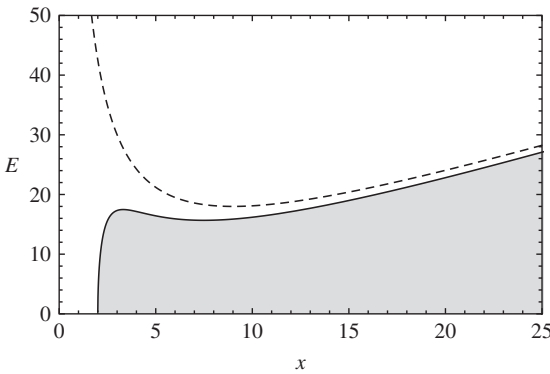

(d) $\Omega=0, B=0$

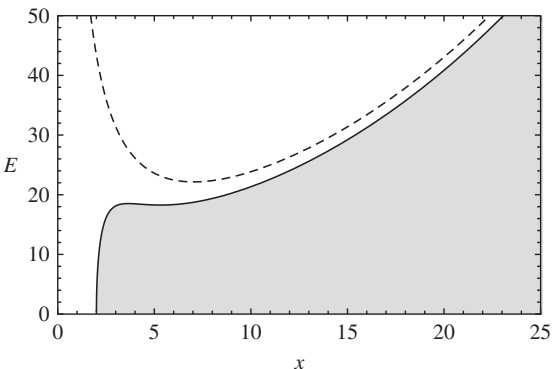

(g) $\Omega=1 / \sqrt{2}, B=0.1$

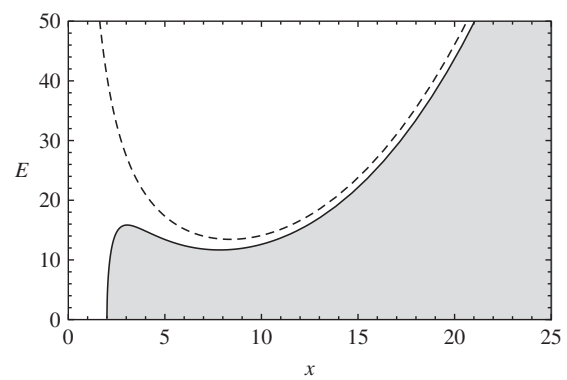

(b) $\Omega=-1 / \sqrt{2}, B=0.2$

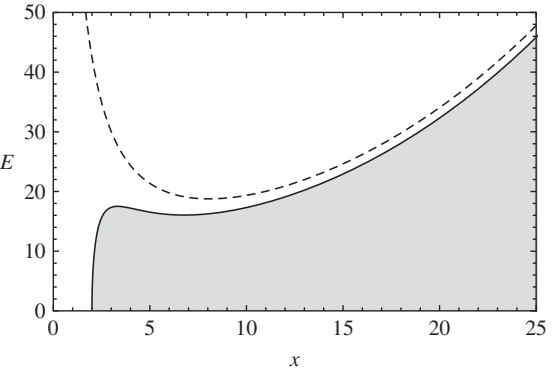

(e) $\Omega=0, B=0.1$

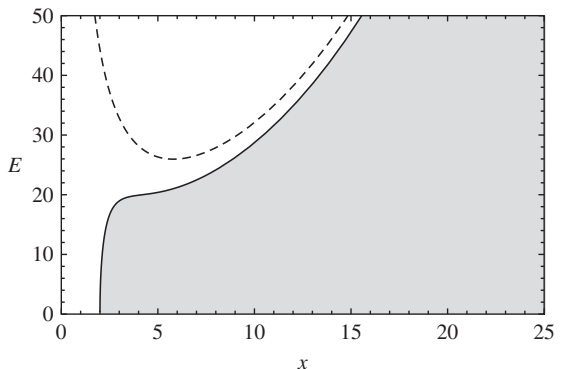

(h) $\Omega=1 / \sqrt{2}, B=0.2$

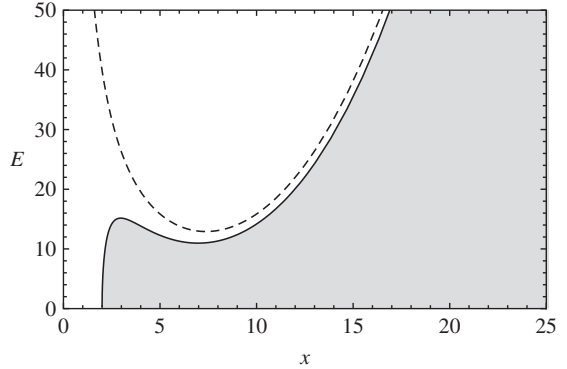

(c) $\Omega=-1 / \sqrt{2}, B=0.3$

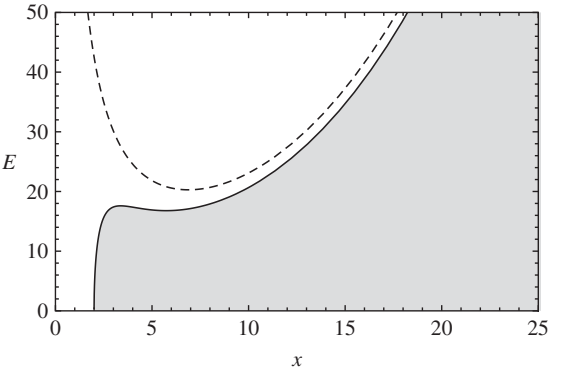

(f) $\Omega=0, B=0.2$

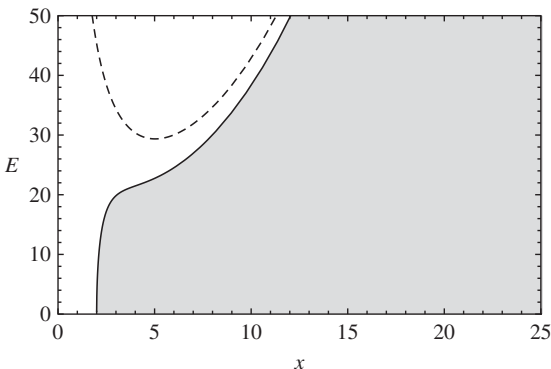

(i) $\Omega=1 / \sqrt{2}, B=0.3$

FIG. 7. Boundary energy $E_{b}$ as a function of the $x$ coordinate for the fixed value of string parameter $J=9$ and different values of magnetic parameter $B$ and current parameter $\Omega$ in the equatorial plane $y=0$. Solid lines correspond to the boundary energy in the Schwarzschild spacetime, while dashed lines to the boundary energy in flat spacetime. The forbidden region of motion is shaded. 

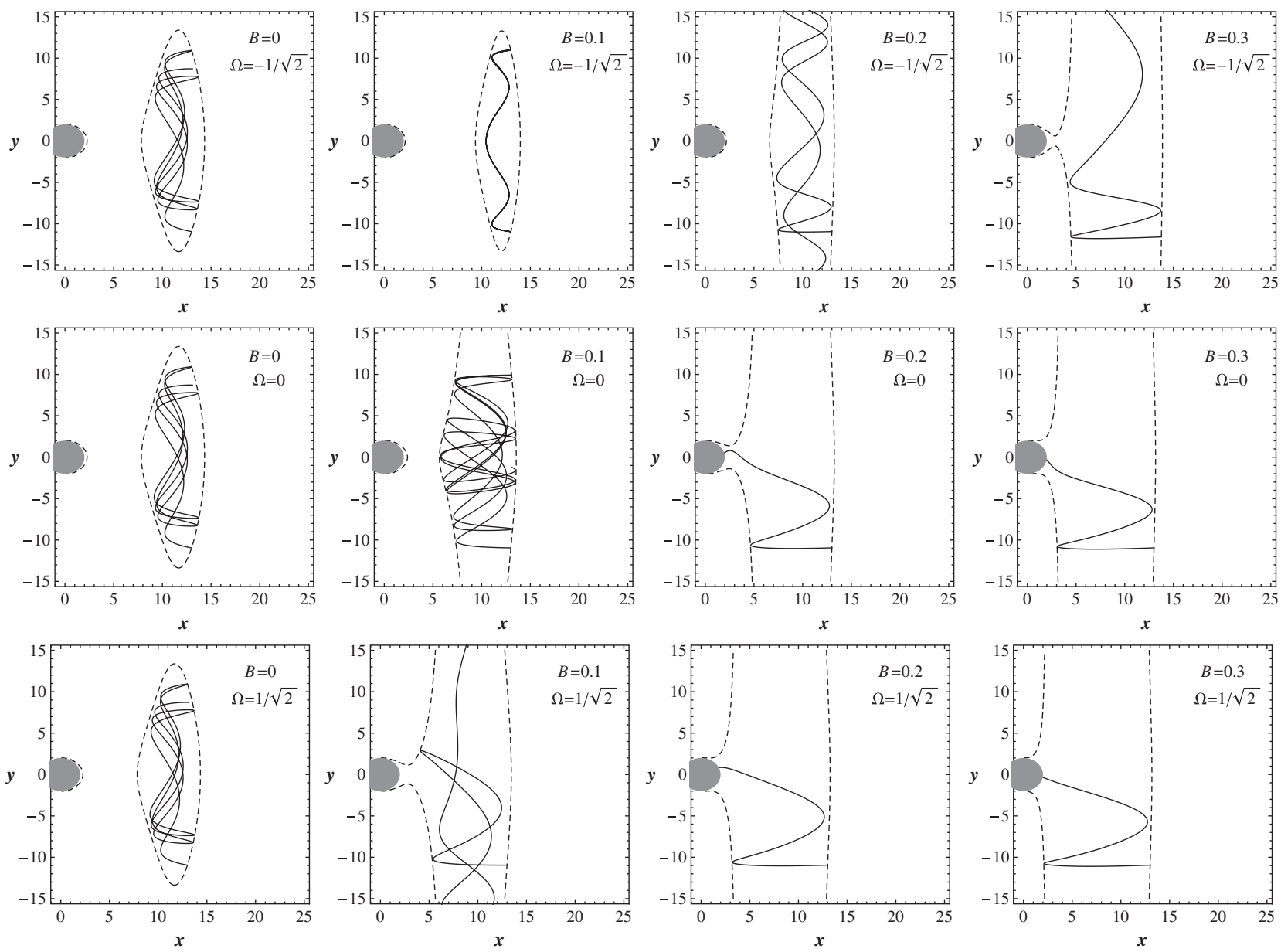

FIG. 8. Boundaries of the motion and numerical simulations of the corresponding trajectories of string loop for the fixed value of the angular momentum parameter $J=12$. The string starts motion from the initial position with coordinates $x_{0}=13, y_{0}=-11$.

momentum parameter is represented in Fig. 2 as the intersection point of the solid thick and the dotted curves. The $x$ profile of the boundary energy function for the different values of the magnetic $B$ and current $\Omega$ parameters are represented in Fig. 7.

\section{String trajectory in the magnetic field}

In Fig. 8 the boundaries of the motion and the numerical simulation of the corresponding trajectories of the string loop for the fixed value of the angular momentum parameter $J=12$ and the different values of magnetic field and electric current are presented. In the first row $\Omega=-\frac{1}{\sqrt{2}}$, in the second row $\Omega=0$, and in the third row $\Omega=\frac{1}{\sqrt{2}}$; the magnetic field takes the values $B=0, B=0.1, B=0.2$, and $B=0.3$. The boundaries of the string motion (dashed curves) are obtained as solutions of the equation of the energy boundary function (42). The trajectories of the string loop motion (solid curve) inside the boundaries are obtained by the integration of the equations of motion (59) and (60). The string starts motion from the initial position with coordinates $x_{0}=13, y_{0}=-11$. For each parameter $\Omega$ the representation starts from the nonmagnetic case when $B=0$. As one can see from the first column there is no difference between these plots due to the absence of the term responsible for the interaction between the magnetic field and electric current. For the different values of the magnetic field and the same initial conditions, one can obtain different types of the motion of the string loop. This confirms the fact that depending on the value of the magnetic field one can change the type of string loop motion, e.g., from trapping into escaping to the infinity or capturing by the black hole.

\section{CONCLUSION}

We have studied the influence of the external uniform magnetic field on the dynamics of an electric currentcarrying string loop moving axisymmetrically on the background of the Schwarzschild black hole. Equations of string motion and the relevant effective potentials in the presence of magnetic field were studied.

It has been shown that the string loop itself is a source of the magnetic field but contribution of the self-magnetic field to the energy of the string motion is negligible being proportional to the square of the self-field. However, this 
field has been taken into account as the interaction between global and local fields as the second term of the effective potential of the string motion using introduced string parameters.

Using the effective potential we have obtained an equation for the energy boundaries of the string loop motion which includes the energy of the electromagnetic background, string fluid energy depending on the angular momentum of the string loop, and the energy given by the Lorenz force which can be associated as the interaction between the magnetic field and the electric currentcarrying loop and can be either positive or negative. To the better understanding of the effect of a magnetic field on string motion, we have illustrated the cases for the absence of a magnetic field along with the cases for different values of magnetic field parameter and different values of the current for relevant results.

It is shown that in the case of the negative current there is a location in spacetime where the electromagnetic field energy can be balanced with the Lorenz force energy. Below this location the effect of the magnetic field will decrease the oscillations of string loop while after this location the oscillations of string loop will increase. By considering a number of examples and various plots of the relevant functions, the possible types of string motion were identified. We have also found the boundaries of the angular momentum parameter in dependence on both the magnetic field and electric current which minimum helped us to identify the possible types of the string loop motion. It is possible to change the type of string loop motion by changing the value of the magnetic field. The most relevant from an astrophysical point of view is the possibility of the escape of string loop to infinity. Because of this reason, we have investigated the flat spacetime limit as the condition of the escape. It was shown that the total energy of the string can be decomposed in the Cartesian components and $x$ component of the energy providing the energy of string oscillations can be equalized to zero which means that string oscillations vanish. In that case the total energy of the string loop will be equal to the $y$ component of the decomposed energy which is responsible for the linear motion of the string loop in the $y$ direction; in such cases the acceleration of the string loop will be maximal. We plan to study acceleration of the jets in dependence on the external magnetic field in a forthcoming paper.

For the configuration under study the magnetic field is orthogonal to the plane of the string motion and it will effect the string oscillation in the $x$ direction only. However no string motion or acceleration in the $y$ direction due to magnetic field is expected. This is contrary to the effect of the repulsive cosmological constant [3] when the string is effected also in the $y$ direction mainly when it crosses the static radius [42-44].

\section{ACKNOWLEDGMENTS}

The authors would like to express their acknowledgements for the Institutional support of the Faculty of Philosophy and Science of the Silesian University at Opava, the internal student grant of the Silesian University SGS/23/2013 and EU grant Synergy CZ.1.07/ 2.3.00/20.0071. This research is supported in part by Projects No. F2-FA-F113 and No. FE2-FA-F134 of the UzAS and by the ICTP through the OEA-PRJ-29 projects. B. A. acknowledges the German Academic Exchange Service (DAAD) and the ENSF, Trieste, Italy travel grants. B. A. and A. T. greatly acknowledge the hospitality at the Max-Planck-Institut für Gravitationsphysik, Potsdam.
[1] T. Jacobson and T. P. Sotiriou, Phys. Rev. D 79, 065029 (2009).

[2] M. Kološ and Z. Stuchlík, Phys. Rev. D 82, 125012 (2010).

[3] Z. Stuchlík and M. Kološ, Phys. Rev. D 85, 065022 (2012).

[4] Z. Stuchlík and M. Kološ, J. Cosmol. Astropart. Phys. 10 (2012) 008

[5] V. Semenov, S. Dyadechkin, and B. Punsly, Science 305, 978 (2004).

[6] M. Christensson and M. Hindmarsh, Phys. Rev. D 60, 063001 (1999).

[7] V. S. Semenov and L. V. Bernikov, Astrophys. Space Sci. 184, 157 (1991).

[8] C. Cremaschini and Z. Stuchlík, Phys. Rev. E 87, 043113 (2013).

[9] H. C. Spruit, Astron. Astrophys. 102, 129 (1981).

[10] A. L. Larsen, Classical Quantum Gravity 11, 1201 (1994).

[11] A. V. Frolov and A. L. Larsen, Classical Quantum Gravity 16, 3717 (1999).
[12] V. P. Frolov, Phys. Rev. D 85, 024020 (2012).

[13] M. Yu. Piotrovich, N. A. Silant'ev, Yu. N. Gnedin, and T. M. Natsvlishvili, arXiv:1002.4948.

[14] I. M. McHardy, E. Koerding, C. Knigge, P. Uttley, and R. P. Fender, Nature (London) 444, 730 (2006).

[15] V. P. Frolov and A. A. Shoom, Phys. Rev. D 82, 084034 (2010).

[16] A. R. Prasanna, Riv. Nuovo Cimento Soc. Ital. Fis. 3, 1 (1980).

[17] J. Kovár, Z. Stuchlík, and V. Karas, Classical Quantum Gravity 25, 095011 (2008).

[18] J. Kovár, O. Kopáček, V. Karas, and Z. Stuchlík, Classical Quantum Gravity 27, 135006 (2010).

[19] S. M. Wagh and N. Dadhich, Phys. Rep. 183, 137 (1989).

[20] L.-X. Li, Phys. Rev. D 61, 084016 (2000).

[21] G. Preti and F. de Felice, Phys. Rev. D 71, 024009 (2005).

[22] M. de Kool, G. V. Bicknell, and Z. Kuncic, Pub. Astron. Soc. Aust. 16, 225 (1999). 
[23] J. M. Miller, J. Raymond, A. Fabian, D. Steeghs, J. Homan, C. Reynolds, M. van der Klis, and R. Wijnands, Nature (London) 441, 953 (2006).

[24] M. Yu. Piotrovich, N. A. Silant'ev, Yu. N. Gnedin, and T. M. Natsvlishvili, Astrophys. Bull. 66, 320 (2011).

[25] A.L. Larsen, Classical Quantum Gravity 10, 1541 (1993).

[26] R. M. Wald, Phys. Rev. D 10, 1680 (1974).

[27] A. L. Larsen, Phys. Lett. A 170, 174 (1992); Mod. Phys. Lett. A 07, 2913 (1992).

[28] A. Vilenkin and E.P.S. Shellard, Cosmic Strings and Other Topological Defects (Cambridge University Press, Cambridge, England, 1994).

[29] D. Garfinkle and C. M. Will, Phys. Rev. D 35, 1124 (1987).

[30] L. Pogosian and T. Vachaspati, Phys. Rev. D 60, 083504 (1999).

[31] D. N. Page, Phys. Rev. D 60, 023510 (1999).

[32] J.P. De Villiers and V.P. Frolov, Classical Quantum Gravity 16, 2403 (1999).
[33] M. Snajdr, V. Frolov, and J.P. De Villiers, Classical Quantum Gravity 19, 5987 (2002).

[34] V. P. Frolov and D. V. Fursaev, Phys. Rev. D 63, 124010 (2001).

[35] M. Snajdr and V. Frolov, Classical Quantum Gravity 20, 1303 (2003).

[36] V. P. Frolov and K. A. Stevens, Phys. Rev. D 70, 044035 (2004).

[37] J. A. Petterson, Phys. Rev. D 10, 3166 (1974).

[38] D. M. Chitre and C. V. Vishveshwara, Phys. Rev. D 12, 1538 (1975).

[39] B. B. Ahmedov, Astrophys. Space Sci. 331, 565 (2011).

[40] I. G. Moss, Phys. Rev. D 83, 124046 (2011).

[41] P.A.R. Ade et al. (Planck Collaboration), arXiv:1303.5085.

[42] Z. Stuchlík, Bull. Astron. Inst. Czech. 34, 129 (1983).

[43] Z. Stuchlík and S. Hledík, Phys. Rev. D 60, 044006 (1999).

[44] Z. Stuchlík and J. Kovář, Int. J. Mod. Phys. D 17, 2089 (2008). 